Images du travail, travail des images

$2 \mid 2016$

Les ouvriers et la photographie : de 1945 à nos jours

\title{
Grand entretien avec Gérald Bloncourt
}

Henri Eckert et Gérald Bloncourt

\section{OpenEdition}

\section{Journals}

Édition électronique

URL : https://journals.openedition.org/itti/1231

DOI : 10.4000/itti.1231

\section{Éditeur}

Université de Poitiers

\section{Référence électronique}

Henri Eckert et Gérald Bloncourt, " Grand entretien avec Gérald Bloncourt », Images du travail, travail des images [En ligne], 2 | 2016, mis en ligne le 01 septembre 2016, consulté le 24 juillet 2021. URL : http://journals.openedition.org/itti/1231 ; DOI : https://doi.org/10.4000/itti.1231

Ce document a été généré automatiquement le 24 juillet 2021.

Images du travail, travail des images 


\title{
Grand entretien avec Gérald Bloncourt
}

\author{
Henri Eckert et Gérald Bloncourt
}

Gérald Bloncourt est né en 1926 en Haïti. Étudiant, il est l'un des leaders des journées insurrectionnelles - restées dans l'histoire haïtienne sous le nom de «Cinq glorieuses de janvier $1946 »-$ qui aboutiront à la chute du pouvoir dictatorial d'alors. Mais, il est expulsé par la junte militaire qui a succédée à la dictature et gagne la France la même année, après un passage par la Martinique. Il s'installe alors à Paris où il entame une carrière de photographe tout en poursuivant ses activités de peintre-graveur et d'écrivain. Il publie, en 2004, un livre intitulé Le regard engagé et sous-titré Parcours d'un franc-tireur de l'image, dans lequel il raconte sa fuite d'Haïti et sa carrière de photographe à Paris. Ses images sont présentées notamment dans le livre Les prolos, publié en 2004 également, qu'il réalise en collaboration avec Mehdi Lallaoui, auteur des textes, et Le Paris de Gérald Bloncourt, paru en 2010. Dans ce dernier ouvrage, Gérald Bloncourt donne à voir un Paris populaire, loin des clichés habituels. En 2013 est présentée, au Musée de l'histoire de l'immigration, l'exposition Pour une vie meilleure, photographies de Gérald Bloncourt, qui regroupe une cinquantaine de photographies en noir et blanc sur l'immigration portugaise en France, réalisées entre 1954 et 1974 (créée au Portugal en 2008 et présentée en divers lieux par la suite). Il expose, en 2014, une série de photographies du monde ouvrier dans le cadre du festival Filmer le travail, à l'Espace Mendès-France à Poitiers. Il a publié aussi, avec Michael Löwy en 2007, Messagers de la tempête, sous-titré André Breton et la révolution de janvier 1946 en Haïti, où les deux auteurs reviennent sur les circonstances des journées de 1946, et fait paraitre, en 2013, son Journal d'un révolutionnaire.

Je demande d'abord à Gérald Bloncourt «comment il en est arrivé à photographier des ouvriers ou plus généralement des individus au travail ».

Gérald Bloncourt: Je ne crois pas que je sois «venu » à la photographie. J'étais graveur et peintre, j'ai été un des fondateurs du Centre d'Art en Haïti et j'ai participé au mouvement qui a débouché sur l'insurrection de 1946. J'ai été chassé d'Haïti la même année et, quand je suis arrivé en France, je n'avais en main que la gravure et la peinture. J'ai collaboré avec une galerie d'art qui voulait que je dessine des péniches : 
ils étaient fous amoureux de mes gouaches, ils voulaient que je ne fasse plus que ça. Et moi j'en ai eu tellement marre de leur fournir trois dessins par semaine que je faisais ça de mémoire dans ma chambre, tu vois! Bon, je gagnais un peu ma croute comme ça, j'ai aussi débarqué des cageots aux Halles de Paris. Par hasard, j'ai trouvé un job dans une boite de photographie, qui s'appelait pompeusement Les éditions photographiques universitaires. Elle avait des contrats avec l'Éducation nationale. On faisait des groupes scolaires, avec la grosse chambre 18x24, le pied, le voile noir, la poire pour : «Souriez!». On alignait les gosses, le professeur. Moi ça m'emmerdait beaucoup et, au bout de quelques mois, j'ai dit au patron: "J'arrête !». Alors, pour me garder, il m'a envoyé photographier les vitraux de la cathédrale de Chartres. J'y ai passé six mois à faire la bascule ${ }^{1}$, parce qu'on était au sol! Oui, il fallait calculer tout ça. Là j'ai appris vraiment le métier de photographe. Après, comme j'étais militant communiste et syndicaliste à l'époque, j'ai provoqué une grève dans la boîte. J'ai été viré mais j'ai été récupéré par L'Humanité, qui avait entendu parler d'un jeune photographe, membre du Parti communiste : j'ai été invité à venir à L'Huma. Je leur ai dit: "Toutes les photographies existent, la photo de sport, de mode, la photo scientifique, le portrait, tu vois, et même la photo pornographique! Mais le photojournalisme je connais pas!» On m'a répondu : «Tu verras! Tu apprendras !».

J'ai ainsi fait mes premières armes à L'Humanité. À l'époque, on photographiait les grèves. On alignait tous les ouvriers devant l'usine, en rang d'oignons et on faisait la photo! La plupart souriaient comme si c'était une chose heureuse. Ça faisait la une de L'Humanité. J'ai dit: "Ce n'est pas possible, il faut montrer leurs cicatrices, leurs souffrances, leurs colères, leurs blessures ». D'instinct je suis allé dans cette direction. Il a fallu que je me batte ! Une fois j'avais été faire un chantier de bâtiment en grève ; de colère les ouvriers avaient écrit sur la palissade, à la craie, "Chantier en grève de $24 \mathrm{~h}$ ». Avec tellement de colère que la craie avait pété à certains coins. Je fais la photo, je la ramène : «Où sont les hommes dans cette photo? » J'ai manqué d'être viré ! J'ai tenu bon et un type qui s'appelait Jean-Pierre Chabrol (devenu un grand écrivain) s'est écrié : « Mais c'est un chef d'œuvre ! C'est ça qu'il faut passer ! ». C'est à partir de là que j'ai commencé à imposer un autre style d'image à L'Huma. Les choses ont changé, nous avions simplement rejoint les Cartier Bresson, les Willy Ronis et tous ces créateurs qui faisaient déjà ce genre d'images dans d'autres revues. Mais à l'époque nous ne regardions même pas cette presse dite «bourgeoise "! J'ai été nommé responsable politique du service photographique et nous avons pu orienter L'Humanité dans cette direction. Je continuais à peindre clandestinement... Pourquoi clandestinement? Parce que Aragon qui avait créé la Maison de la Pensée Française, où j'ai connu Alain Guérin ${ }^{2}$, et plein de gens importants, imposait le « réalisme socialiste ». Comme j'écrivais aussi - j'étais jeune poète - j'y allais! Je me suis rendu compte qu'Aragon, qui prônait ce fameux réalisme socialiste, avec Fougeron ${ }^{3}$ et d'autres estimaient que ce qui m'attirait était de l'art décadent. Ils ne touchaient pas à Picasso, parce que Picasso donnait du fric au parti. Mais les jeunes peintres devaient peindre le maçon avec la truelle à la main, etc., faire de l'art soviétique quoi ! Je n'étais pas d'accord, je me suis insurgé. Mais comme je ne voulais pas être en désaccord officiellement avec le parti qui, pour moi, était le seul porteur des espoirs de changements de la société, de la révolution, je me suis écrasé. Je peignais clandestinement. Je trouvais par contre que la photographie était un moyen 
« réaliste socialiste» qui permettait de participer, de dénoncer. J'ai pris cette direction en me disant que je serai le Gromaire ${ }^{4}$ de la classe ouvrière.

Gérald Bloncourt s'éloigne du Parti communiste après les événements de Hongrie et travaille alors comme photographe reporter indépendant pour différents journaux, hormis « la presse réactionnaire de droite » précise-t-il. II participe à la fondation de l'Association nationale des journalistes reporters photographes et cinéastes (ANJRPC) avec Roger Pic, Willy Ronis et d'autres photographes. Association qui jouera un grand rôle pour la défense des droits des reporters. À cette époque il crée les Editions murales. II s'est agi, en somme, d'organiser des expositions itinérantes : " J'ai fait plus de 500 expositions, et à l'intérieur de ces expositions, je poussais les animateurs à faire venir des conférenciers dans ces centres culturels, les MJC, les comités d'entreprises, à présenter des livres et à les diffuser. C'était extraordinaire. » Gérald Bloncourt poursuit: «Par la suite, comme j'étais militant haïtien, j'ai tout abandonné pour rentrer dans mon pays quand la dictature est tombée. À mon retour en France, ça n'existait plus. Les comités d'entreprise vendaient des billets de théâtre, des voyages, ils ne faisaient plus d'animation culturelle parce qu'avec les horaires, il n'y avait plus d'exposition dans les locaux... L'activité culturelle était en fait, en panne. J'ai donc abandonné ce type de démarches devenues désuètes.

Mais il me reste des souvenirs magnifiques! Par exemple, à Saint-Nazaire, j'avais exposé L'homme du quotidien, c'est-à-dire une quarantaine de photos avec des textes, collés sur les panneaux. Plus de 3000 ouvriers sont venu voir. J'en avais la chair de poule. J'étais au milieu d'eux, ils ne me connaissaient pas. Ils se disaient entre eux : «T'as vu, regarde cette photo, y'a ça, y'a ça... » Ils me faisaient découvrir mes propres images. Ce sont eux qui m'ont appris mon métier, m'ont obligé à réfléchir. Je me suis dit que la photo était un moyen de communication visuelle entre les hommes. J'ai aussi compris que la presse était ma salle d'exposition quotidienne, permanente, populaire, ouverte au public de la rue, à l'homme de tous les jours. J'ai pris conscience que c'était un moyen extraordinaire pour participer, pour contribuer à dénoncer les injustices, pour les faire connaître. Je me suis aussi rendu compte qu'un photojournaliste était un transmetteur de mémoire. Qu'il en avait la possibilité et la responsabilité !

\section{Faire des photos : l'art, la technique et l'émotion}

La discussion porte ensuite sur deux photos publiées dans Les prolos, qui représentent des mineurs au travail dans une mine de glaise à Provins, en 1961. 
Photographie 1. Mineur de glaise à provins - 1961. La force de travail !

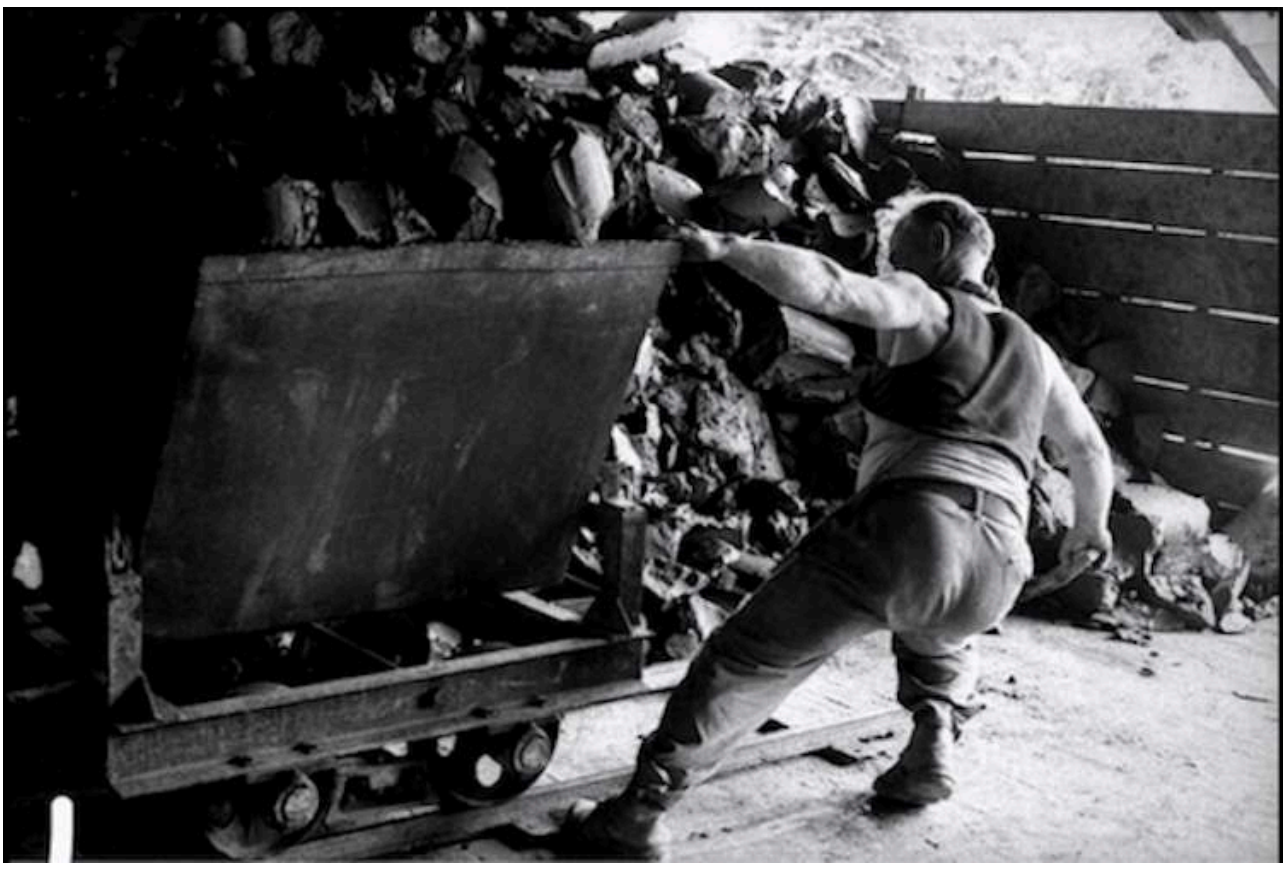

(c) Gérald Bloncourt.

Photographie 2. Mineur de glaise à Provins - 1961.

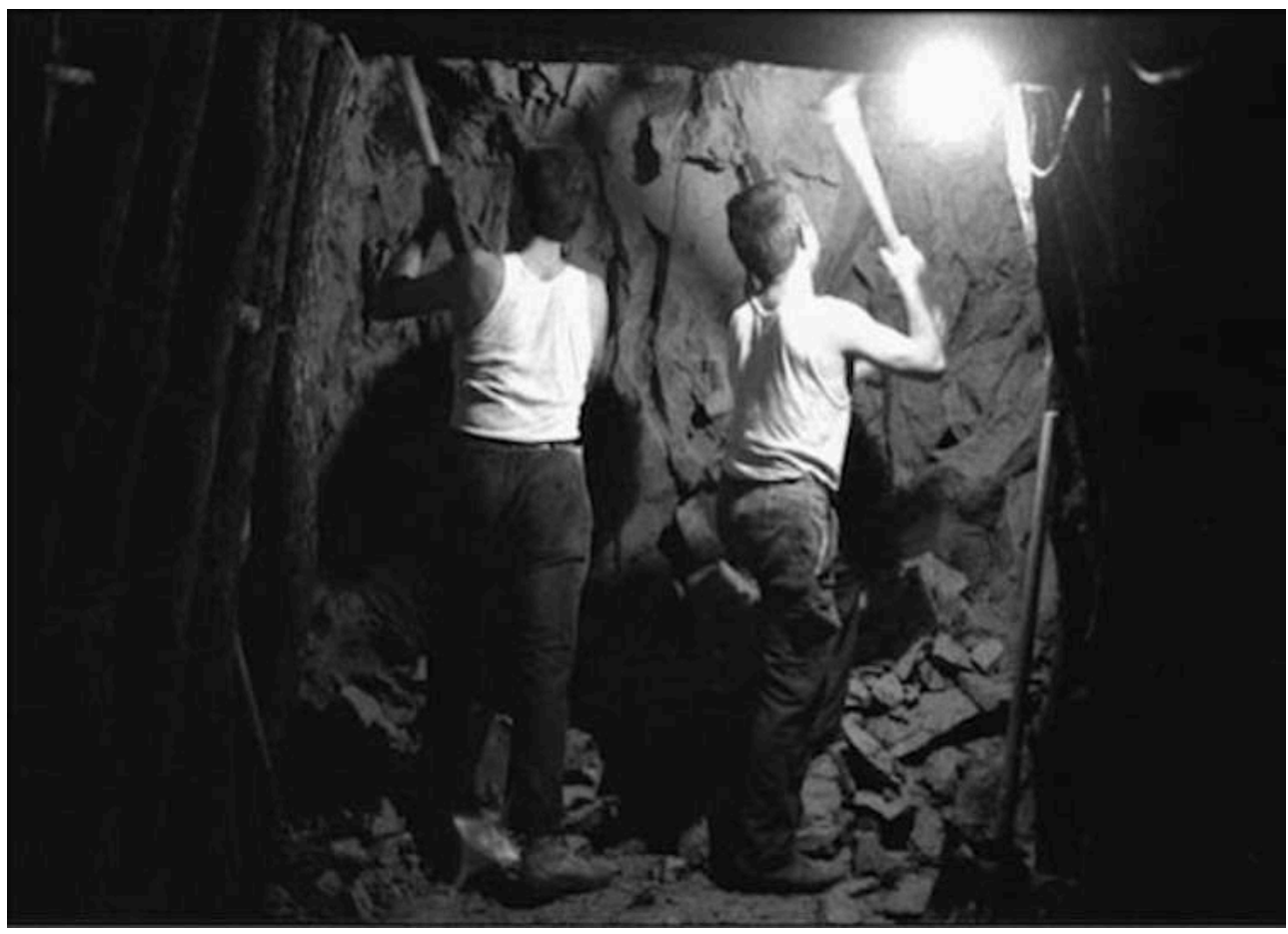

Ici pas de silicose à craindre ! Juste l'humidité qui pénètre jusqu'à la moelle des os... Chaque coup de pioche harassant... chaque ampoule aux mains... chaque feuille de paye dérisoire...

(c) Gérald Bloncourt.

Gérald Bloncourt : On m'avait signalé une mine de glaise, je me suis dit qu'est-ce que c'est que ça? J'ai fait les mines de charbon, les mines de fer, de phosphate. Mais de 
glaise... Je vais donc à Provins. J'y découvre effectivement une mine de glaise. Ici, pas de crainte d'explosion de grisou ou de silicose, mais l'humidité. Les rhumatismes! J'ai passé deux journées là bas. Dans une des galeries de la mine, j'ai vu cet homme qui tirait un chariot, de toutes ses forces, à la main ! J'ai fait cette photo et après j'ai parlé avec le gars ; il touchait le smic ! C'était un immigré polonais. En faisant cette image j'ai immédiatement pensé à Marx. J'ai d'ailleurs intitulé ma photo : «La force de travail»!

" Nous allons donc, en même temps que le possesseur d'argent et le possesseur de force de travail, quitter cette sphère bruyante où tout se passe à la surface et aux regards de tous, pour les suivre tous deux dans le laboratoire secret de la production, sur le seuil duquel il est écrit : No admittance except on business [On n'entre pas ici, sauf pour affaires !]. Là, nous allons voir non seulement comment le capital produit, mais encore comment il est produit lui-même ». (Karl Marx, Le Capital, Livre 1, Section II, Chapitre VI, « Achat et vente de la force de travail », p. 135, Éditions sociales).

Ce métier est quelquefois mystérieux. Au moins le temps compris entre la prise de vue et le développement de la pellicule. On n'est jamais sûr d'avoir réalisé une image parfaite. Il faut attendre cet instant où elle t'est révélée. Alors ou tu t'engueule ou tu crie de joie pour l'avoir parfaitement réussie.

La photographie ça commence d'abord là. Bloncourt pointe son doigt sur son front, sans doute pour indiquer le cerveau ! Et il rajoute: Donc tu penses ta photo, tu la construit en un éclair avec ton cerveau qui donne l'ordre à ton doigt d'appuyer sur le déclencheur de l'appareil. II faut que celui-ci soit bien solidement dans tes mains et que ton doigt soit au bon endroit pour appuyer au bon moment. C'est une gymnastique. On est des athlètes de haut niveau. Mais tant que ce n'est pas développé tu n'es jamais sûr d'avoir vraiment réussi ! Je parle de tout ce que doit contenir l'image, l'expression, le regard, la révélation de ce que tu as voulu transmettre. Maintenant, avec le numérique, tu le vois très vite, immédiatement. L'avantage du numérique, c'est que tu peux doubler, tripler la prise de vue. Avec la pellicule tu étais limité. Tu avais 12 photos pour un rollefleix, tu en avais 36 dans un rouleau pour un $24 \times 36$. Tu étais limité. Avec les plaques c'était encore pire, tu avais deux magasins de douze plaques pour faire une étape du Tour de France... Et si tu te trompais de vingt centimètres c'était flou. Bobet s'était échappé ? Paf, tu avais mis dix centimètres de trop et c'était flou. J'en ai sorti des photos de Bobet. Tu vois, c'est un métier! On est les chauffeurs routiers du regard!

QUESTION : Quand tu photographiais Bobet, tu pensais à quelqu'un qui était au travail ?

Gérald Bloncourt : Hum... Difficile de répondre. J'ai fait pas mal d'étapes du Tour de France. Tout le monde en parle, à la radio on parle de ça, dans le journal, tu finis par connaître ces champions et tu finis par les aimer! Je me disais que je suis chargé de transmettre leurs exploits à des millions d'yeux qui ne me connaissent pas, qui ne connaissent pas mon nom. Il faut leur montrer leur idole, en plein effort. C'est une mission, il faut que je fasse plaisir. Ce n'est évidemment pas la même démarche de classe, comme avec la photo de « la force de travail ». C'était autre chose... Tu fais ta photo, il est beau, il est en plein effort et tu sais qu'elle va être publiée et que les types vont admirer, même ceux qui ne font pas de cyclisme. Brassens disait : « Quand je fais une chanson, si dans une chaumière ou dans un HLM, une ménagère est heureuse, j'ai touché au but ». Moi c'était pareil! 
QUESTION : Et celle-là (la deuxième photo de mineurs de glaise)?

Oui, ce sont d'autres mineurs. J'ai dû en faire plusieurs. Mais ces deux-là, c'est Medhi ${ }^{5}$ il les a choisies. Il aurait pu en prendre d'autres... Mais eux, tu vois, ils sont côte à côte, ils bossent ensemble et après, quand ils auront terminé ils iront casser la croute ensemble... Et puis ils rentreront, chacun de son côté. Peut être qu'il y a un qui a une femme avec des enfants. Je pense à ça quand je fais la photo, à leur vie. Je me sens responsable. Je me sens responsable et donc j'ai du respect, de l'amitié. Quelquefois de l'amour, ça arrive... La petite gosse des mineurs de Trieux... Il y avait tous les jours des meetings : la tribune, les responsables syndicaux, le curé, le maire, tout le monde était là ! Et tous les jours je la croisais, à l'abri de la main de son père. Une fillette que sa maman avait bien habillée, une belle petite écharpe de laine. Je l'ai aperçue, je n'ai pas tout de suite fait attention... Elle me regardait, elle ne me quittait pas des yeux. J'allais, je venais, elle me regardait, je la fascinais, j'avais trois appareils autour du cou, je n'étais pas habillé en mineur, j'étais l'étranger. Elle me regardait. Et puis, d'un seul coup, je me suis dit : mais c'est ça la grève ! Le type, il se bat pour qui ? Pour son avenir à elle, c'est ça la grève ! Alors j'ai fait un gros plan. Elle est à l'abri de la main de son père, elle me regarde et j'ai choisi comme légende La grève de Trieux, 1963. Quand je suis revenu à La Vie Ouvrière, avec mes photos, j'ai dit : celle-là, c'est une pleine page. Ils m'ont dit... Il faut montrer les mineurs... J'ai dit: écoutez, en montrant une autre photo, celle là par exemple, vous pouvez la mettre en petit, en disant qu'ils étaient 10 000, vous mettez ce que vous voulez... Mais celle là, c'est la pleine page avec comme légende : Trieux, la grève, 1963. Ils l'ont finalement passée en pleine page. La semaine d'après, au courrier des lecteurs, ils étaient débordés. Les gens étaient émus par cette photo. Et là j'ai gagné en autorité, Ça m’a permis d'imposer mon regard... Ce qui a changé le leur d'ailleurs, dans l'utilisation des photos. Mais je te disais, il y avait des noms prestigieux à La vie ouvrière. Il y avait Willy Ronis, Cartier-Bresson, Doisneau, Roquer Pic, Janine Niépce, Boubat... C'est à cette époque que la photographie a gagné ses lettres de noblesse. 
Photographie 3. Grève des mineurs de Trieux - Mineurs de fer (Lorraine) - 1963.

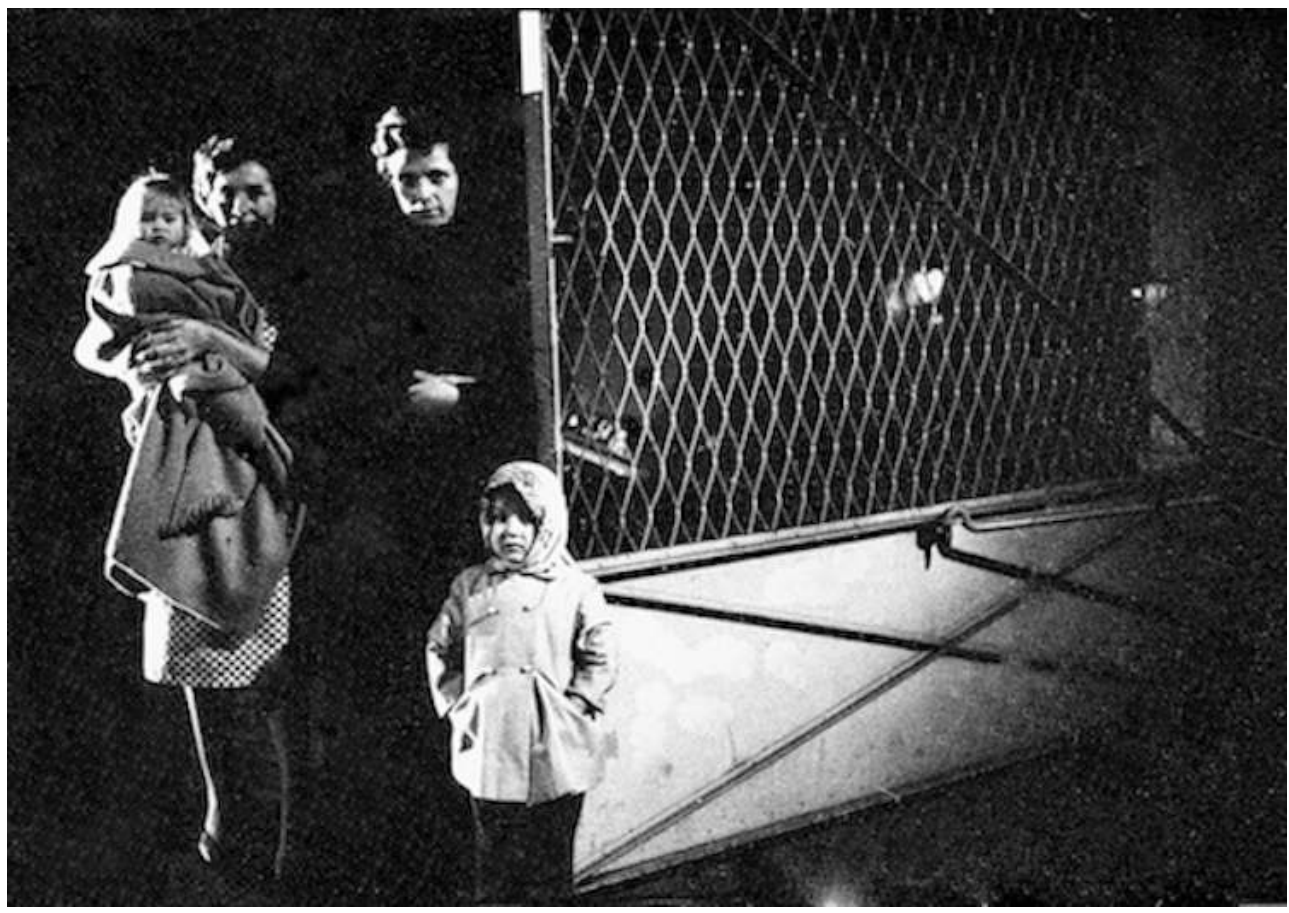

Les hommes occupent la mine. Les femmes viennent dans la nuit, aux nouvelles. II fait froid. C'est l'hiver.

(c) Gérald Bloncourt. 
Photographie 4. Grève de mineurs de fer de Trieux - 1963.

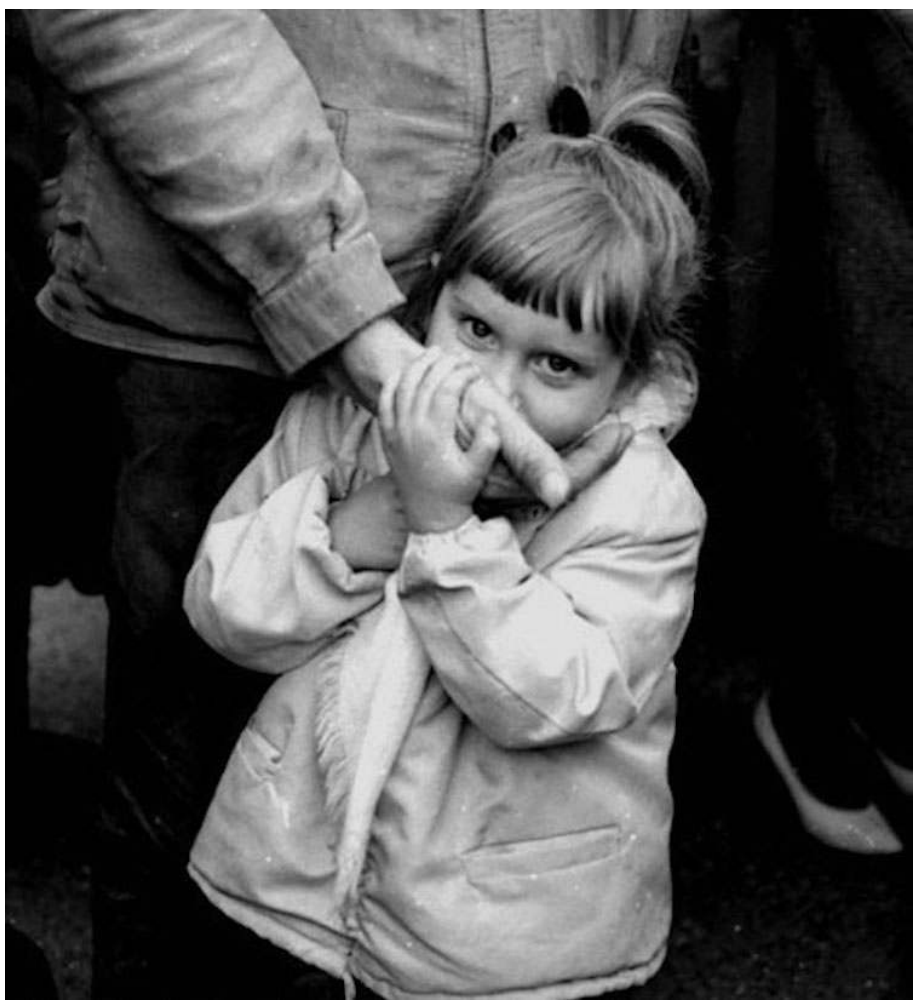

Tous les jours au meeting qui avait lieu sur la Place du village, il y avait cette petite fille à l'abri dans la main de son père.

(c) Gérald Bloncourt.

\section{L'usine, entrer dans l'usine...}

La discussion s'oriente vers la photographie à l'usine : I'usine, comment y entrer ? Comment entrer dans «le laboratoire secret de la production » comme disait Marx? 
Photographie 5. En Moselle à l'usine Gerlasch à Bouzonville - Sidérurgie - 24/5/1973.

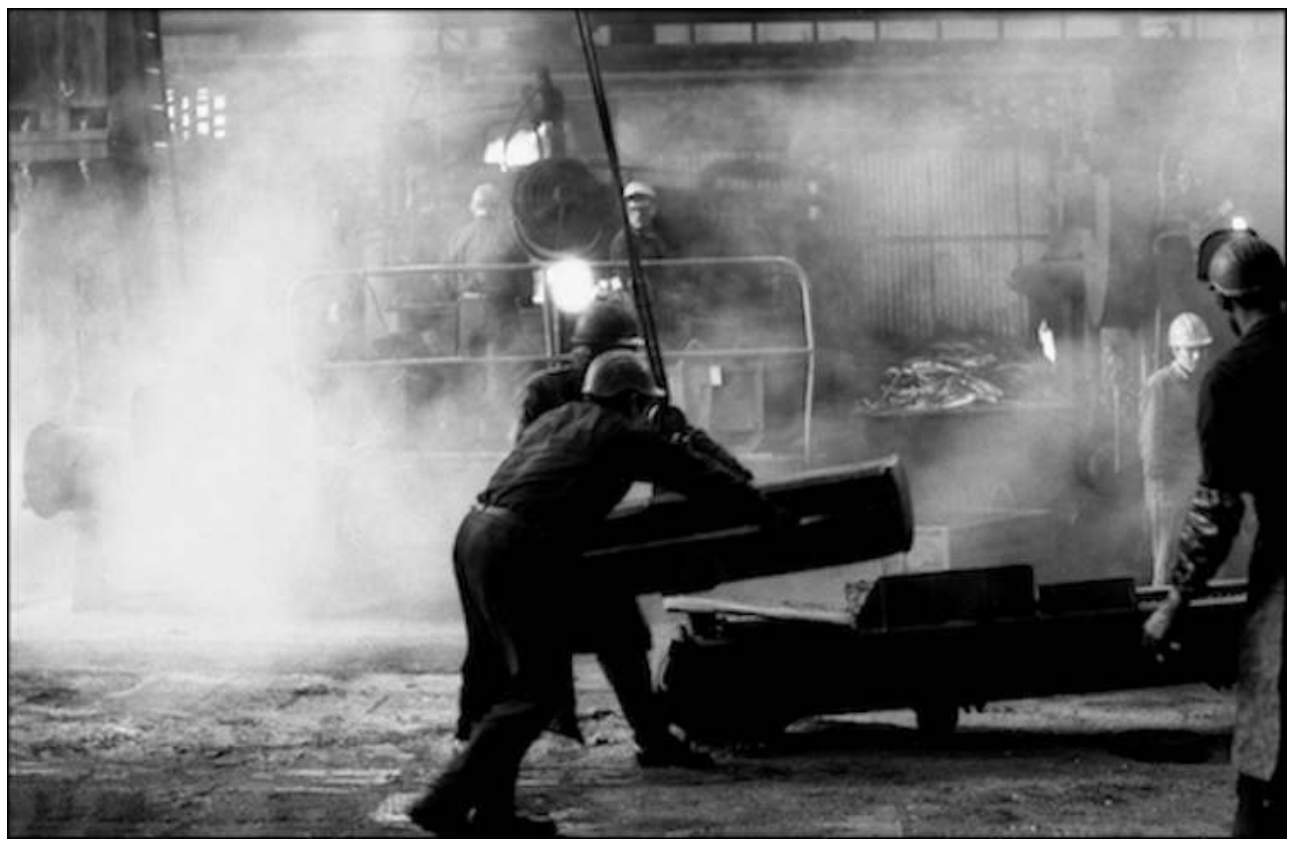

(c) Gérald Bloncourt

Photographie 6. Usines Gerlach à Bouzainville - 25/5/1973.

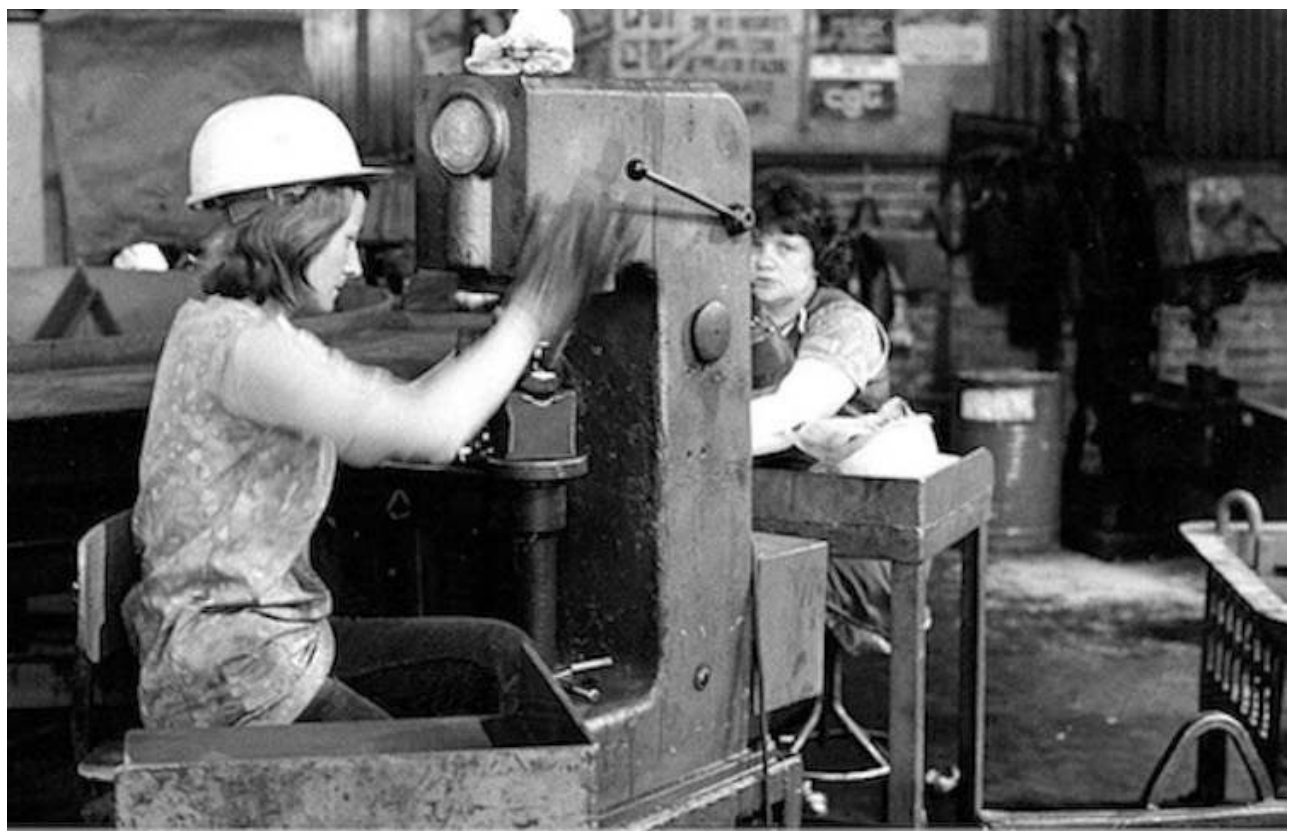

(C) Gérald Bloncourt.

QUESTION : Tu as pu entrer dans l'usine...

Gérald Bloncourt : Bien sûr... J'ai réussi à me faufiler ! On m'a foutu à la porte mais j'ai quand même fait des photos! Y'a cette espèce d'atmosphère, ce sont des fumées nocives d'ailleurs. Ils n'ont pas de masque mais ils travaillent là-dedans. Et l'effort... On sent toute cette chaleur, tu vois, et ils sont obligés d'être habillés pour ne pas être trop brulés. J'ai fait des photos de parties de corps brulées. Il (Mehdi Lallaoui) a choisi celle-là à cause de la force qu'il faut mettre en œuvre pour guider la charge. C'était à 
l'usine de fonte de Bouzonville, c'est une scène que j'ai capturé dans l'usine, une de ces scènes où j'essaie de situer les choses, pour transmettre aux autres l'émotion que j'ai eu devant cette image. J'aurais pu cadrer uniquement sur l'action. Mais là en cadrant plus large, je montrais l'ambiance de l'usine, les petits détails. Il y a les vitres tout en haut, tu vois, même ça, ça compte : pour montrer la grandeur de l'usine. Alors que si tu coupes là (Gérald Bloncourt cache la partie haute de la photo), tu ne vois plus. Là ça montre l'usine... Mais ça c'est une déformation professionnelle. Il faut du talent (Gérald Bloncourt éclate de rire), je dis ça modestement, on s'habitue à cadrer, et on pense... En faisant, ça va très vite, c'est de l'instantané, la photo c'est un centième de seconde, un millième, donc il faut que le cerveau travaille. Et au moment où tu fais ta photo, il y a tout ça qui vient, c'est une forme de gymnastique. Tout ça se passe en une fraction de seconde, la fraction de seconde qui t'est nécessaire pour faire la photo. Que ton cerveau dise au doigt: appuie! Mais tout rentre dans une photo, y compris des choses qui t'échappent. Et les types quand ils la regardent, ils montrent ça à leurs copains en insistant sur des détails qui t’avaient échappés, et toi tu découvres aussi ta photo! J'essaye de te transmettre ce type de réflexion qui m'habite. Ici c'est pareil. Là, il faisait assez obscur, je ne pouvais pas faire un instantané rapide... Voilà, pour obtenir l'image, il me fallait descendre au dixième de seconde et, au dixième de seconde tu risque de bouger. Ici il y a l'autre ouvrière, qui est de face, qui jette un coup d'œil vers moi... Le fait qu'elle me regarde prouve qu'elle est vivante, elle se rend compte que quelqu'un est là. J'aime bien. J'essaie d'analyser... Bon j'aurais peut-être pu faire mieux, on peut toujours faire mieux !

La compagne de Gérald Bloncourt, qui passe par là et a entendu ses propos : "Les gens te regardent souvent. Elle, elle te regarde. C'est-à-dire que les gens, ils se donnent parfois à photographier. Ils ne font pas une photo comme ça, il y a un échange avec le photographe».

Gérald Bloncourt: Moi je trouve que ça donne tout une profondeur. Parce que la photo... Je sais pas ce que tu en penses mais on voit cette scène qui se passe dans une usine, on voit ces deux femmes au travail et puis ce qui était un peu sur un plan, ça prend une profondeur, une troisième dimension quoi. C'est pour ça que je l'aime beaucoup celle là ! [Puis, revenant à la première des deux photos...] Là on est dans l'usine, on sent que c'est une usine immense, les fenêtres et l'action au fond de l'atelier, tout ça te montre qu'il y a un espace gigantesque, tout un univers. Quand les ouvriers arrivent le matin, après s'être lavés et être sortis de chez eux, ils viennent travailler, ils rentrent dans cet univers. J'ai une autre série de photos, des types dans l'univers sidérurgique, si tu vas sur mon blog, tu pourras les voir. Là il faut la multiplicité des photos pour montrer les différents... Quand tu as tout ça sous les yeux, tu rentres avec eux dans leur univers. Parce qu'il y a ça aussi, une photo ça ne peut pas tout dire. Quelquefois ça dit beaucoup mais quelquefois, quand il y a une série d'images, l'une vient épauler l'autre... C'est le jeu de la mise en page. Le texte aussi, le texte terriblement important, la légende de la photo. La légende de la photo et le journal qui la donne à voir. La même photo dans deux journaux différents peux dire le contraire mais... Elle peut dire des choses différentes dans un cas et dans l'autre. Celui qui fait l'image n'est pas tout à fait maître de son image! Moi je me battais avec mes journaux, des journaux que j'adorais, La Vie Ouvrière, des magazines, etc. Je les connaissais, j'y allais! Mais il y a des journaux plus distants, par exemple L'Express. Je n'avais aucun rapport direct sinon de temps en temps, comme ça. Tu donnes ta photo, elle est prise mais après elle est utilisée... Je me battais quand même 
pour que soit respecté l'esprit de ma légende, qu'on n'aille pas dire quelque chose de négatif, contraire à ce que je voulais dire. En général, ils l'ont toujours respecté ; mais en fonction du contexte de l'article, mon cher, en fonction de la légende... L'objectivité n'existe pas! Moi je ne suis pas objectif, j'ai pris parti. Quand je prends une photo, j'essaie de traduire mes sentiments et celui qui va l'utiliser aussi... L'objectivité, en tant que telle, n'existe donc pas comme ça. Il n'y a rien de moins objectif qu'un objectif photographique (Gérald Bloncourt rit). Ça m'est venu comme ça, j'ai écrit cette phrase un jour. C'est toi, c'est vous autres qui vous intéressez à la photo, de faire avancer tout ça. Vous avez du pain sur la planche... Toi comme moi, nous voulons changer la société, que ça aille mieux, tu comprends, mais c'est une bataille et ce que je viens de te dire fait partie de cette bataille.

QUESTION : Mais pour rentrer dans les usines, comment tu faisais ?

Gérald Bloncourt : Renault Billancourt, par exemple! J'étais de mèche avec les gars du comité d'entreprise, j'ai mis un bleu, l'appareil 24x36 dans une poche, deux objectifs dans l'autre poche. Je rentrais entouré par les gars. L'un se mettait devant moi, je rentrais dans l'usine comme ça. En général j'étais guidé : les copains syndiqués me disaient d'aller par ici, dans tel atelier... J'y allais mais je faisais gaffe au contremaitre qui surveillait, fallait souvent se planquer. Derrière une machine, dans les pissotières, etc. Il fallait parfois attendre... J'ai fait ça je sais pas combien de fois. Un jour je suis rentré guidé par un militant de la CGT, lorsqu'il a vu le contremaitre il s'est tiré, je me suis retrouvé tout seul. Je me suis planqué mais quand le contremaître est parti, j'étais perdu dans l'usine, je ne savais plus comment faire. Alors j'ai fait des photos et puis il a fallu ressortir. Mais même la sortie, je savais pas où elle était. Il y a des gars qui m'ont regardé bizarre : tu connais pas la sortie ? Alors j'ai dit: «Écoute, je suis photographe à La Vie Ouvrière, CGT ». «Ah ouais, ben moi je suis de la CFTC » qu'il m'a répondu le gars. Et il a poursuivi : «Alors bah, ça fait rien, t'es un copain quand même ! » et il m'a emmené. « Tiens, viens! » Je suis passé au nez et à la barbe des gardiens et je suis sorti de l'usine. Ca ne m'est arrivé qu'une fois. Mais d'autres fois, je suis rentré et je me suis fait virer !

Une autre fois, Antoinette ${ }^{6}$ m'avait demandé de photographier les ouvrières de chez Hutchinson. J'y vais, j'arrive à l'usine, des grandes grilles... Face au gardien qui me demande ce que je veux, je réponds que je suis journaliste, reporter et photographe et que je voudrais rentrer dans l'usine pour faire des photos. "Attendez, bougez pas!» Il téléphone, arrive un gars des bureaux, un responsable, derrière la grille toujours fermée qui me demande à son tour ce que je veux. Je lui dis que je suis journaliste reporter, que je fais des images, ma spécialité c'est le monde du travail et que j'ai donc besoin de rentrer dans l'usine pour faire des photos. «Pour qui vous travaillez? » «Ben je travaille pour le journal Antoinette, le journal des femmes de la CGT. » «Dehors, dehors! » qu'il a gueulé. Deux gardiens sont arrivés, j'ai fichu le camp... Antoinette était une revue mensuelle, j'avais du temps, plusieurs jours devant moi. J'avais repéré une ligne de chemin de fer qui passait à-côté de l'usine, qui dominait l'usine. Le lendemain je loue un téléobjectif: ils venaient de sortir un $1000 \mathrm{~mm}$ qui faisait 30 centimètres de longueur, assez large, avec des miroirs à l'intérieur, un seul pied suffisait pour le supporter. J'y colle mon 24x36 et je monte sur la voie ferrée d'où je plonge dans l'usine, dans la cours de l'usine. Il y avait des garages à vélo dans cette cour, il faisait gris mais, à un moment, un petit rayon de soleil vient taper au milieu de la cour. Et je vois un beau gars, un ouvrier, et une jolie 
femme, une ouvrière. Ils arrivent, se placent juste dans le rayon de soleil et se font un baiser sur la bouche. Alors là je fais la photo et puis une trentaine d'autres, les ouvrières en train d'accrocher les vélos, de pointer. Il y avait aussi une sorte de baie vitrée, on voyait les machines à l'intérieur... J'ai fait tout ce que j'ai pu et je ramène le tout au journal. Ils étaient heureux comme tout! Il y avait alors un metteur en page qui s'appelait Jean-Pierre Jouffroy, qui est devenu un grand critique d'art, qui dit: «Magnifique! » en voyant la photo des deux amoureux. Il la publie en pleine page! La revue sort. Et voilà que je reçois un coup de téléphone de la rédactrice en chef, Louisette Blancard, qui me dit: «Viens vite, viens vite, c'est très important! " Je prends ma voiture, j'arrive, elle me dit : « C'est incroyable, la photo des amoureux fait un scandale.» Le type était syndiqué à la CGT et la fille, son mari était soldat en Algérie, par ailleurs syndiqué à la CFTC. «Les camarades ont pris la chose très mal, ils veulent faire interdire la publication.» La diffusion a été stoppée sur tout le département. Les syndicats CGT et CFTC se sont réunis et ont calmé le jeu. Voilà une histoire qui m'est arrivée, tu vois (rires) !

Une autre fois chez Renault : Jean Rabaté 7 me demande d'aller à Flins, photographier les ateliers de peinture au pistolet, où les ouvriers travaillaient sans masque. [...] Comment faire pour entrer? Au téléphone ils ne vont pas me donner l'autorisation. Il faut forcer le passage. Je connaissais des types des deux syndicats et j'essaie de prendre contact, mais ils me disent : « Là on peut pas t'aider, des fois on peut mais là on peut pas.» Bon, j'y vais au culot. Je prends un pied, je prends une chambre photographique $13 \times 18$ pour être bien vu, mes sacoches de photographe et j'arrive à l'entrée. Les gardiens me regardent, ils ne me demandent rien : je rentre! Ben écoute, si tu n'es pas autorisé, tu n'arrives pas comme ça si tu n'est pas autorisé !... Ils m'ont donc laissé passer. Je rentre dans l'usine. Je marche et je me rends compte qu'on commence... il y a des gardiens, des contremaitres qui commencent... Je me sens repéré quand même. Je prends mon appareil, j'ouvre le pied, je fais semblant de faire de la mise au point mais avec mon $24 \times 36$ je prends toutes les photos que je peux. Je marche, je marche ... Je flaire, et j'arrive, la fumée, la vapeur... Je tombe sur la peinture au pistolet. Je pose mon gros appareil. Je fais les photos avec le petit. Mais fallait ressortir. Mais si je ressors comme ça d'un seul coup... Je me dit ce qui a marché à l'entrée va marcher au retour. Mais non, il y a deux types qui viennent et me demandent: "Qu'est-ce que vous faites là?» «Vous voyez bien, je photographie!» «Mais vous avez une autorisation? " "Mais bien sûr, sinon je ne serais pas là.» «Ben montrez là! " «Je suis autorisé ! " Mais par qui ? " Mais par moi! » Qu'est-ce que tu voulais que je fasse d'autre? «Suivez nous!» Alors les gars m'emmènent dans des bureaux, avec tout mon matériel, puis chez un responsable, un chef. Celui-ci me dit: "Qu'est-ce que vous faites dans l'usine sans autorisation? Vous ne savez pas que c'est dangereux, faut demander des autorisations. " J'ai répondu : «Ah bon, je savais pas moi, je suis journaliste, je pensais que je pouvais... » Je jouais l'idiot! «Et qu'est-ce que vous faites comme photos?» «Ben je photographie les gens au travail.» Il me dit: «Mais pour qui travaillez-vous?» «Ben je suis indépendant, mais là c'est pour l'Humanité.» «Quoi !? Bon, ben écoutez, on va vous donner deux types qui vont vous accompagner, parce qu'il faut pas aller n'importe où. » Il me dit: "Bon ça va, on va vous donner deux types et on mange dans une heure, vous revenez, vous déjeunez avec nous!» Surréaliste... Deux types qui m'accompagnent et m'aident à porter mes trucs. Je n'ai que mon petit $24 \times 36$. Ils me font faire le tour de l'usine, les chaines de montages... [...] Rabbaté me téléphone : 
«Mon cher, génial, comment tu as fait pour ramener tout ça? C'est formidable! Parce que nous, on a envoyé trois types avant toi, ils se sont fait éjecter tous les trois. »

\section{Légendes...}

La discussion porte à présent sur une photo dont le titre est Le poids de la monnaie.

Photographie 7. Le poids de la monnaie - 1965.

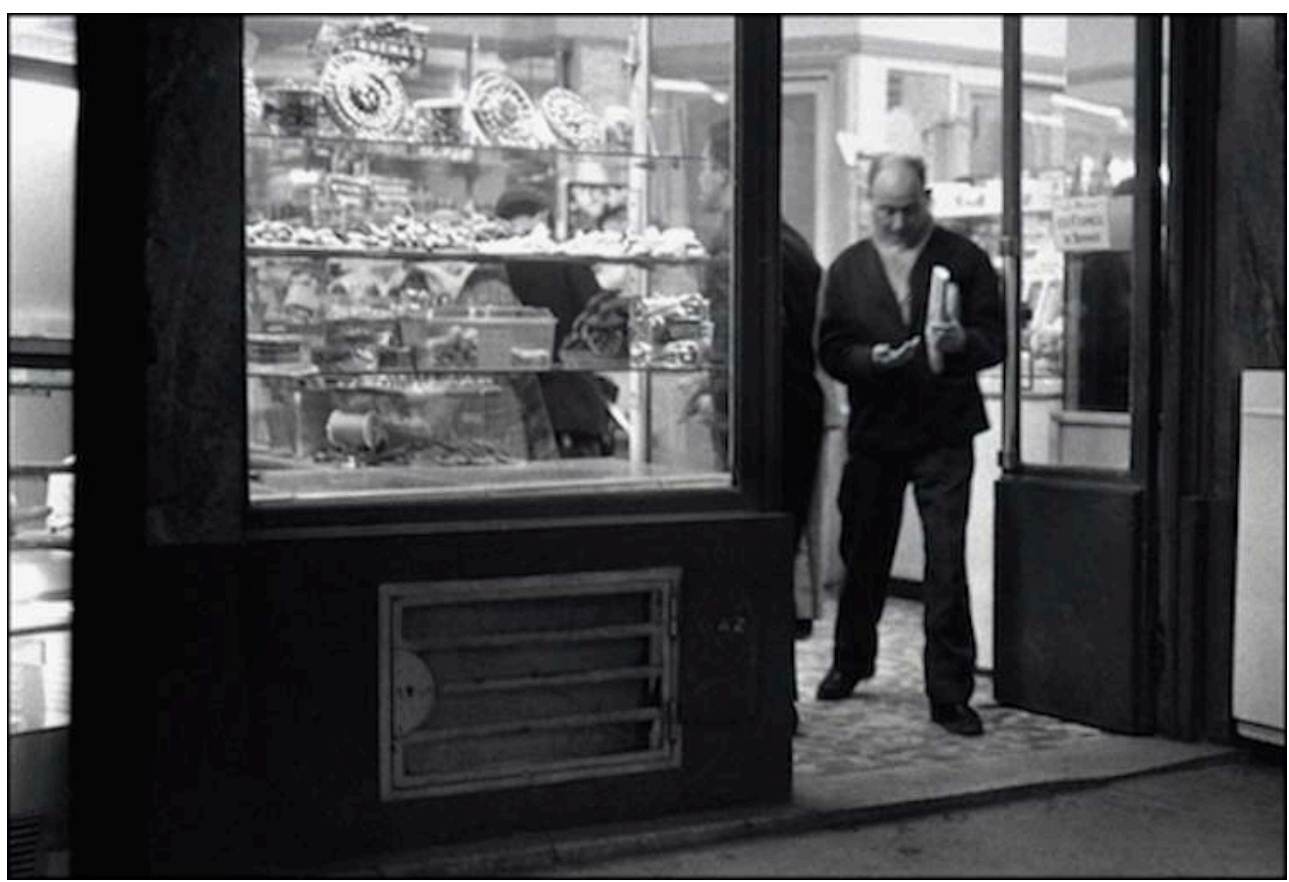

Ouvrier immigré portugais travaillant chez Citroën à Paris, achetant son pain dans une boulangerie de quartier de l'usine, près de la place Balard.

(c) Gérald Bloncourt.

Gérald Bloncourt : Le poids de la monnaie c'est ça? Ecoute, j'étais chez Citroën. Ça, c'est de mon initiative. J'allais de nuit devant des usines où le matin à l'embauche. J'ai une série de photos de l'embauche à Citroën. Et là il y avait une boulangerie, et le gars, avant d'entrer à l'usine, il achète son pain. Parce qu'il va casser sa croûte, parce que, à l'usine, ils ont leur gamelle. Et là il est en train de vérifier sa monnaie. Parce qu'une pièce d'un centime c'est une pièce. C'est important, c'est le poids de la monnaie, donc la légende ça coule de source. La légende est fiancée à la photographie!

C'est important, les pièces c'est important, les gars ils vivaient encore au jour le jour, ils avaient de petites payes, hein! C'est gigantesque. Quand j'ai fait ça j'étais content, j'étais heureux, j'ai dégusté ma photo... C'est le type de chance, de la chance voulue, parce que j'étais dans ma voiture, je regardais un peu ce qui se passait et puis il y avait cette boulangerie et d'un seul coup j'ai vu le type rentrer, je me suis dit: tiens ça c'est surement un ouvrier, il va acheter son pain et paf, $j$ 'ai fait la photo en pensant au poids de la monnaie. Et puis après je suis descendu de voiture, j'ai parlé avec lui. C'était un portugais, pour l'authentification de ma photo. Ça aurait pu être un 
français, mais c'était un portugais. Je vérifie quand même! Le coiffeur dans le bidonville par exemple, c'est le bidonville portugais, là il y a pas besoin de demander.

Photographie 8. La petite portugaise du bidonville - 1969.

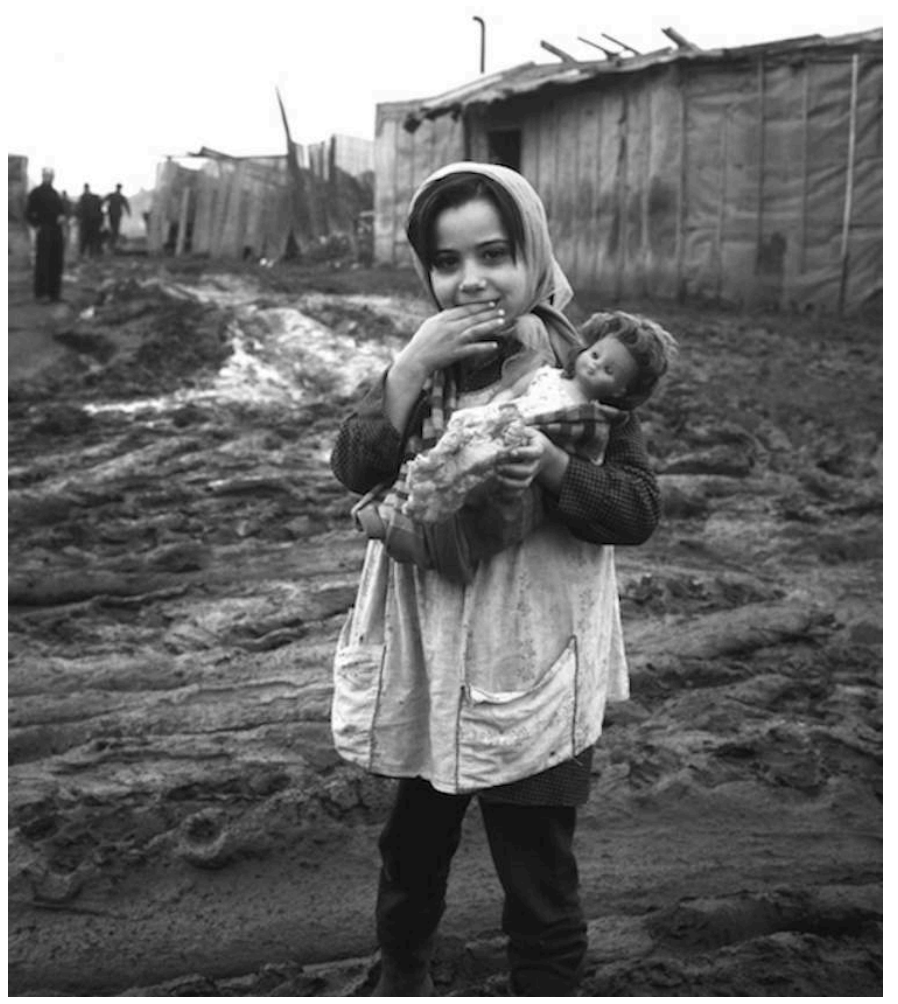

Bidonville à Saint-Denis - région parisienne. Petite portugaise immigrée. Elle s'appelle Maria da Conceição Tina Melhorado et m'a retrouvé 46 après la prise de cette photo!

(c) Gérald Bloncourt.

Tu sais, la petite fille du bidonville, elle m'a retrouvé 47 ans après. Elle doit avoir plus de 55 ans. Elle est venue me voir. Son histoire est extraordinaire. J'y allais comme je voulais dans le bidonville de Saint-Denis, on me connaissait, je faisais les photos, j'étais connu. Ce jour-là, il me restait une photo sur mon rouleau, j'avais terminé, je n'avais pas d'autre pellicule. Il me restait une photo dans mon Rolleiflex et j'allais partir. J'avais mangé avec des portugais, je les saluais, et je vois cette petite fille qui m'attendait, parce qu'elle me connaissait. Elle m'avait vu plusieurs fois, et elle m'attendait pour que je la photographie. Elle avait son sourire, sa poupée, elle attendait. Je sais que j'ai encore une photo à faire! Clac, je fais la photo. Si je l'avais ratée, il n'y aurait pas eu d'histoire. Plus tard, j'expose au Portugal, au musée d'art moderne de Lisbonne: ils ont trouvé la photo tellement belle qu'ils en ont fait la couverture d'un livre et réalisé une affiche. Elle était dans tout Lisbonne, partout, et les amis de cette gosse, qui la connaissaient, qui avaient vécu dans le bidonville de Saint-Denis l'ont reconnue : c'est toi! Elle m'envoie un e-mail, qui me dit: je pense que ça pourrait être moi. Alors on a fait une enquête, je demande le nom, le prénom, à quel moment, quelle date, je préviens des amis portugais de continuer l'enquête, et c'est bien elle! Elle est venue me voir, il y avait un cinéaste qui était là. Je l'ai fait venir quand j'ai exposé au musée de l'immigration à Paris. J'ai dit : « Vous l'invitez. » Ils m'ont répondu : «Ah oui mais le budget... » J'ai dit : «Si vous l'invitez pas, je ne viens pas. » Alors ils l'ont invitée. Je l'avais vue deux ou trois fois, je la connaissais. 
Donc j'arrive, elle était là. Elle a logé chez moi pendant deux jours. Il y avait un tas de journalistes, dont des américains. Parce que le musée de l'immigration, ça intéresse ! Dès qu'elle est arrivée, ils nous ont mis ensemble, ils nous ont photographiés, et puis après ils m'ont complètement oublié, c'était elle la vedette. Je lui ai dit : «Faut que tu écrives ton histoire. " Il y a un an, elle a sorti son livre. Elle y raconte son histoire. J'en ai écrit la préface.

Je suis un homme heureux (éclat de rire)!

\section{NOTES}

1. Entendre à utiliser un objectif à bascule et décentrement, qui permet de corriger les perspectives en photo d'architecture.

2. Alain Guérin, né en 1932, journaliste à L'humanité et poète.

3. André Fougeron (1913-1998), peintre autodidacte, chef de file d'un nouveau réalisme qui l'amènera, après la deuxième guerre mondiale, à s'engager dans la voie du réalisme socialiste.

4. Marcel Gromaire (1892-1971), peintre et graveur français qui a produit une œuvre originale, à l'écart des courants mais à la recherche d'une forme de réalisme inspirée notamment de Fernand Léger.

5. Il s'agir de Mehdi Lallaoui, qui a choisi les photos qui composent Les prolos et rédigé les textes.

6. Antoinette était un magazine mensuel édité par la CGT, à destination d'un lectorat féminin. Le magazine est paru entre 1955 et 1989.

7. Jean Rabaté est journaliste à L'humanité.

\section{AUTEURS}

\section{HENRI ECKERT}

Professeur de sociologie à l'Université de Poitiers et membre du GRESCO. 